\title{
Best separable approximation with semi-definite programming method
}

\author{
M. A. Jafarizadeh ${ }^{a, b, c}$, M.Mirzaee ${ }^{a, b} \dagger$ M.Rezaee $^{a, b} \ddagger$ \\ ${ }^{a}$ Department of Theoretical Physics and Astrophysics, Tabriz University, Tabriz 51664, Iran. \\ ${ }^{b}$ Institute for Studies in Theoretical Physics and Mathematics, Tehran 19395-1795, Iran. \\ ${ }^{c}$ Research Institute for Fundamental Sciences, Tabriz 51664, Iran.
}

August 6, 2018

\footnotetext{
*E-mail:jafarizadeh@tabrizu.ac.ir

${ }^{\dagger}$ E-mail:mirzaee@tabrizu.ac.ir

${ }^{\ddagger}$ E-mail:karamaty@tabrizu.ac.ir
} 


\begin{abstract}
The present methods for obtaining the optimal Lewenestein- Sanpera decomposition of a mixed state are difficult to handle analytically. We provide a simple analytical expression for the optimal Lewenstein-Sanpera decomposition by using semidefinite programming. Specially, we obtain the optimal Lewenstein-Sanpera decomposition for some examples such as: Bell decomposable state, Iso-concurrence state, generic two qubit state in Wootters's basis, $2 \otimes 3$ Bell decomposable state, $d \otimes d$ Werner and isotropic states, a one parameter $3 \otimes 3$ state and finally multi partite isotropic state. Keywords: Optimal Lewenstein-Sanpera decomposition, Semi-definite programming, Bell decomposable states, Werner and isotropic states.

PACs Index: 03.65.Ud
\end{abstract}




\section{INTRODUCTION}

Entanglement is one of the most striking features of quantum mechanics $[1,2]$. In the case of pure states it is easy to check whether a given state is, or is not entangled. For mixed states, however, the statistical properties of the mixture can hide the quantum correlations embodied in the system, making thus the distinction between separable and entangled states enormously difficult.

In the pioneering paper [3], a very interesting description of entanglement was achieved by defining the best separable approximation (BSA) of a mixed state. In the case of 2qubit system, it consists of a decomposition of the state into a linear combination of mixed separable part and a pure entangled one. In this way, the whole non-separability properties are concentrated in the pure part.

In the Ref. [3], the numerical method for finding the BSA has been reported. Some analytical results are also obtained for special states of two qubit states [4]. Further in [5] the BSA of two qubit state has been obtained algebraically. They have also shown that in some cases the weight of the entangled part in the decomposition is equal to the concurrence of the state. An attempt to generalize the results of Ref [3] is made in [6].

There is another method we can use which achieves exactly the same effect, called semidefinite programming(SDP). Over the past years, SDP in particular, have come to be recognized as valuable numerical tools for control system analysis and design. In SDP one minimizes a linear function subject to the constraint that an affine combination of symmetric matrices is positive semidefinite. SDP, has been studied (under various names) as far back as the 1940s. Since 1990 many applications have been discovered in areas such as estimation, signal processing and it is currently considered to be the hottest area in optimization. Although SDP is designed to be applied in numerical methods it can be used for analytical computations. All of the above mentioned applications indicate, that the method of SDP is very useful. Some 
authors try to use the SDP to construct an explicit entanglement witness and entanglement distillation $[7,8,9]$.

In this paper we use the SDP method in order to obtain the optimal Lewenstein-Sanpera decomposition (LSD) of a mixed state. Then we show how to perform the optimal LSD for well known different examples via SDP method.

The paper is organized as follows:

In section-2 we define SDP. In section -3 we give a brief review of Optimal LSD. In section -4, using the SDP method we obtain the optimal LSD of some mixed state density matrices such as: Bell decomposable state, Iso-concurrence state, generic two qubit state in Wootters's basis, $2 \otimes 3$ Bell decomposable state, $d \otimes d$ Werner and isotropic states, a one parameter $3 \otimes 3$ state and finally multi partite isotropic state. The paper is ended with a brief conclusion.

\section{Semi-definite programming}

A SDP is a particular type of convex optimization problem [10]. A SDP problem requires minimizing a linear function subject to a linear matrix inequality (LMI) constraint [11]:

$$
\begin{array}{cl}
\operatorname{minimize} & \mathcal{P}=c^{T} x \\
\text { subject to } & F(x) \geq 0,
\end{array}
$$

where $\mathrm{c}$ is a given vector, $x^{T}=\left(x_{1}, \ldots, x_{n}\right)$, and $F(x)=F_{0}+\sum_{i} x_{i} F_{i}$, for some fixed hermitian matrices $F_{i}$. The inequality sign in $F(x) \geq 0$ means that $F(x)$ is positive semidefinite.

This problem is called the primal problem. Vectors $\mathrm{x}$ whose components are the variables of the problem and satisfy the constraint $F(x) \geq 0$ are called primal feasible points, and if they satisfy $F(x)>0$ they are called strictly feasible points. The minimal objective value $c^{T} x$ is by convention denoted by $\mathcal{P}^{*}$ and is called the primal optimal value.

Due to the convexity of set of feasible points, SDP has a nice duality structure, with, the 
associated dual program being:

$$
\begin{gathered}
\operatorname{maximize} \quad-\operatorname{Tr}\left[F_{0} Z\right] \\
Z \geq 0 \\
\operatorname{Tr}\left[F_{i} Z\right]=c_{i} .
\end{gathered}
$$

Here the variable is the real symmetric (or Hermitean) matrix Z, and the data c, $F_{i}$ are the same as in the primal problem. Correspondingly, matrices Z satisfying the constraints are called dual feasible (or strictly dual feasible if $Z>0$ ). The maximal objective value of $-\operatorname{Tr} F_{0} Z$, i.e., the dual optimal value, is denoted by $d^{*}$.

The objective value of a primal(dual) feasible point is an upper (lower) bound on $\mathcal{P}^{*}\left(d^{*}\right.$. The main reason why one is interested in the dual problem is that one can prove that $d^{*} \leq \mathcal{P}^{*}$, and under relatively mild assumptions, we can have $\mathcal{P}^{*}=d^{*}$. If the equality holds, one can prove the following optimality condition on $x$ :

A primal feasible $x$ and a dual feasible $Z$ are optimal which is denoted by $\hat{x}$ and $\hat{Z}$ if and only if

$$
F(\hat{x}) \hat{Z}=\hat{Z} F(\hat{x})=0
$$

This latter condition is called the complementary slackness condition.

In one way or another, numerical methods for solving SDP problems always exploit the inequality $d \leq d^{*} \leq \mathcal{P}^{*} \leq \mathcal{P}$, where $\mathrm{d}$ and $\mathcal{P}$ are the objective values for any dual feasible point and primal feasible point, respectively. The difference

$$
\mathcal{P}^{*}-d^{*}=c^{T} x+\operatorname{Tr}\left[F_{0} Z\right]=\operatorname{Tr}[F(x) Z] \geq 0
$$

is called the duality gap. If the equality $d^{*}=\mathcal{P}^{*}$ holds, i.e., the optimal duality gap is zero, then we say that strong duality holds. 


\section{Optimal Lewenstein-Sanpera decomposition}

According to pioneering work of Lewenstein and Sanpera [3], any bipartite density matrix $\rho$ has a decomposition of the form

$$
\rho=(1-\lambda) \rho_{e}+\lambda \rho_{s}^{\prime}
$$

where $\rho_{s}^{\prime}$ is a separable density matrix, $\rho_{e}$ is a entangled state, and the parameter $\lambda \in[0,1]$. According to the theorem (2) of reference [3], any 2-qubit density matrix $\rho$ can be written as

$$
\rho=\lambda \rho_{s}^{\prime}+(1-\lambda)|\psi\rangle\langle\psi|
$$

where the entangled part is pure state, but in general $\rho_{e}$ can be a mixed or a pure state where the whole entanglement of $\rho$ is concentrated in $(1-\lambda) \rho_{e}$.

Rare exceptions aside, the LSD of a given (non separable) $\rho$ is not unique, there is usually a continuum of LSD to choose from. The decomposition with the largest weight $\lambda$ of the separable part is the optimal LSD with respect to the chosen separable set, which is proved to be uniquely determined. According to its definition, the separable part of this decomposition is called the best separable approximation (BSA) of $\rho$, and its weight $\lambda$ the separability.

\section{Optimal Lewenstein-Sanpera decomposition with semi- definite programming}

In this section using the SDP method we obtain the optimal LSD of a mixed state for some well known different mixed states. 


\subsection{Optimal Lewenstein-Sanpera decomposition for Bell-decomposable state}

A Bell decomposable (BD) state is defined by:

$$
\rho=\sum_{i=1}^{4} p_{i}\left|\psi_{i}\right\rangle\left\langle\psi_{i}\right|, \quad 0 \leq p_{i} \leq 1, \quad \sum_{i=1}^{4} p_{i}=1
$$

where $\left|\psi_{i}\right\rangle$ is Bell state, given by:

$$
\begin{aligned}
& \left|\psi_{1}\right\rangle=\left|\phi^{+}\right\rangle=\frac{1}{\sqrt{2}}(|\uparrow \uparrow\rangle+|\downarrow \downarrow\rangle), \\
& \left|\psi_{2}\right\rangle=\left|\phi^{-}\right\rangle=\frac{1}{\sqrt{2}}(|\uparrow \uparrow\rangle-|\downarrow \downarrow\rangle), \\
& \left|\psi_{3}\right\rangle=\left|\psi^{+}\right\rangle=\frac{1}{\sqrt{2}}(|\uparrow \downarrow\rangle+|\downarrow \uparrow\rangle), \\
& \left|\psi_{4}\right\rangle=\left|\psi^{-}\right\rangle=\frac{1}{\sqrt{2}}(|\uparrow \downarrow\rangle-|\downarrow \uparrow\rangle) .
\end{aligned}
$$

In terms of Pauli's matrices, $\rho$ can be written as,

$$
\rho=\frac{1}{4}\left(I \otimes I+\sum_{i=1}^{3} t_{i} \sigma_{i} \otimes \sigma_{i}\right)
$$

where [12]

$$
\begin{aligned}
& t_{1}=p_{1}-p_{2}+p_{3}-p_{4}, \\
& t_{2}=-p_{1}+p_{2}+p_{3}-p_{4}, \\
& t_{3}=p_{1}+p_{2}-p_{3}-p_{4} .
\end{aligned}
$$

From the positivity of $\rho$ we get

$$
\begin{aligned}
& 1+t_{1}-t_{2}+t_{3} \geq 0 \\
& 1-t_{1}+t_{2}+t_{3} \geq 0 \\
& 1+t_{1}+t_{2}-t_{3} \geq 0 \\
& 1-t_{1}-t_{2}-t_{3} \geq 0
\end{aligned}
$$


These equations form a tetrahedral with its vertices located at $(1,-1,1),(-1,1,1),(1,1,-1)$, $(-1,-1,-1)[12]$. In fact these vertices denote the Bell states given in Eqs. (4-2) to (4-5), respectively.

On the other hand $\rho$ given in Eq. (4-6) is separable if and only if $t_{i}$ satisfy Eq. (4-8) together with the following equation

$$
\begin{aligned}
& 1+t_{1}+t_{2}+t_{3} \geq 0 \\
& 1-t_{1}-t_{2}+t_{3} \geq 0 \\
& 1+t_{1}-t_{2}-t_{3} \geq 0 \\
& 1-t_{1}+t_{2}-t_{3} \geq 0
\end{aligned}
$$

Inequalities (4-8) and (4-9) form an octahedral with its vertices located at $O_{1}^{ \pm}=( \pm 1,0,0)$, $O_{2}^{ \pm}=(0, \pm 1,0)$ and $O_{3}^{ \pm}=(0,0, \pm 1)$. So, tetrahedral is divided into five regions. Central regions, defined by octahedral, are separable states $\left(p_{k} \leq \frac{1}{2}\right)$. There are also four smaller equivalent tetrahedral corresponding to entangled $\operatorname{states}\left(p_{k}>\frac{1}{2}\right.$ for only one of $\left.k=1, \ldots, 4\right)$, where $p_{k}=\frac{1}{2}$ denote to boundary between separable and entangled region. Each tetrahedral takes one Bell state as one of its vertices.

Now in order to obtain optimal LSD of entangled BD state given in (4-1), with $p_{1}>\frac{1}{2}$, we first choose an arbitrary separable state

$$
\rho_{s}^{\prime}=\sum_{i=1}^{4} p_{i}^{\prime}\left|\phi_{i}\right\rangle\left\langle\phi_{i}\right|, \quad 0 \leq p_{i} \leq 1, \quad \sum_{i=1}^{4} p_{i}^{\prime}=1, \quad p_{1}^{\prime}<\frac{1}{2}, \sum_{i=1}^{4} p_{i}^{\prime}=1
$$

in the separable region. Then using strict SDP optimization prescription of section (2), we try to optimize $\operatorname{Tr}\left(\Lambda \rho_{s}^{\prime}\right)$ with respect to $\rho-\Lambda \rho_{s}^{\prime}>0$, where the feasible solution corresponds to

$$
\Lambda_{\max }=\min \left\{\frac{p_{1}}{p_{1}^{\prime}}, \frac{p_{2}}{p_{2}^{\prime}}, \frac{p_{3}}{p_{3}^{\prime}}, \frac{p_{4}}{p_{4}^{\prime}}\right\}
$$

Now, using the inequalities

$$
\Lambda_{\max } \leq \frac{p_{i}}{p_{i}^{\prime}}, \text { for } i=2,3,4
$$


and summing over the indices $\mathrm{i}=2,3$ and 4 , we obtain

$$
\left(1-p_{1}^{\prime}\right) \Lambda_{\max } \leq\left(1-p_{1}\right)
$$

since, we have

$$
\frac{p_{1}}{p_{1}^{\prime}} \geq \frac{1-p_{1}}{1-p_{1}^{\prime}}, p_{1}>\frac{1}{2} \geq p_{1}^{\prime}
$$

The only possible choice of $\Lambda_{\max }$ which is consistent with the positivity of $\rho-\Lambda_{\max } \rho_{s}^{\prime}$ is

$$
\Lambda_{\max }=\frac{\left(1-p_{1}\right)}{\left(1-p_{1}^{\prime}\right)} .
$$

This choice of $\Lambda_{\max }$ given in (4-15) saturates the inequalities (4-12) and turns the inequalities to equalities, that is, we have $p_{i}^{\prime}=\frac{p_{i}}{\Lambda_{\max }}, i=2,3,4$. The equation (4-15) indicates that $\Lambda_{\max }$ is a monotonic increasing function of $p_{1}^{\prime}$ and its maximum value corresponds to $p_{1}^{\prime}=\frac{1}{2}$, with

$$
\Lambda_{\max }=2\left(1-p_{1}\right)
$$

and

$$
p_{i}^{\prime}=\frac{p_{i}}{2\left(1-p_{1}\right)}, i=2,3,4
$$

Substituting the results that obtained for $\Lambda_{\max }$ and $p_{i}^{\prime}, i=1,2,3,4$ in $\rho-\Lambda_{\max } \rho_{s}^{\prime}$, we obtain

$$
\rho-\Lambda_{\max } \rho_{s}^{\prime}=\left(2 p_{1}-1\right)\left|\phi_{1}\right\rangle\left\langle\phi_{1}\right|
$$

which is a pure state in agreement with theorem (2) of Ref.[3].

Therefore, we have

$$
\rho=2\left(1-p_{1}\right) \rho_{s}^{\prime}+\left(2 p_{1}-1\right)\left|\phi_{1}\right\rangle\left\langle\phi_{1}\right|,
$$

which is optimal LSD of BD states in agreement with [5].

\section{$4.2 \quad$ Iso-concurrence decomposable states}

In this section we define iso-concurrence decomposable (ICD) states, then we obtain optimal LSD for this example. The iso-concurrence states are defined by $[14,15,16]$

$$
\left.\left.\left|\phi_{1}\right\rangle=\cos \theta|00\rangle+\sin \theta|11\rangle\right), \quad\left|\phi_{2}\right\rangle=\sin \theta|00\rangle-\cos \theta|11\rangle\right),
$$




$$
\left.\left.\left|\phi_{3}\right\rangle=\cos \theta|01\rangle+\sin \theta|10\rangle\right), \quad\left|\phi_{4}\right\rangle=\sin \theta|01\rangle-\cos \theta|10\rangle\right) .
$$

It is quite easy to see that the above states are orthogonal and thus span the Hilbert space of $2 \otimes 2$ systems. Now we can define ICD states as

$$
\rho=\sum_{i=1}^{4} p_{i}\left|\phi_{i}\right\rangle\left\langle\phi_{i}\right|, \quad 0 \leq p_{i} \leq 1, \quad \sum_{i=1}^{4} p_{i}=1
$$

These states form a four simplex (tetrahedral) with its vertices defined by $p_{1}=1, p_{2}=1$, $p_{3}=1$ and $p_{4}=1$, respectively.

Peres-Horodeckis criterion $[17,18]$ for separability implies that the state given in Eq. (4-22) is separable if and only if the following inequalities are satisfied

$$
\begin{aligned}
& \left(p_{1}-p_{2}\right) \leq \sqrt{4 p_{3} p_{4} / \sin ^{2} 2 \theta+\left(p_{3}-p_{4}\right)^{2}}, \\
& \left(p_{2}-p_{1}\right) \leq \sqrt{4 p_{3} p_{4} / \sin ^{2} 2 \theta+\left(p_{3}-p_{4}\right)^{2}}, \\
& \left(p_{3}-p_{4}\right) \leq \sqrt{4 p_{1} p_{2} / \sin ^{2} 2 \theta+\left(p_{1}-p_{2}\right)^{2}}, \\
& \left(p_{4}-p_{3}\right) \leq \sqrt{4 p_{1} p_{2} / \sin ^{2} 2 \theta+\left(p_{1}-p_{2}\right)^{2}} .
\end{aligned}
$$

Inequalities (4-23) to (4-26) divide tetrahedral of density matrices to five regions. The central regions, defined by the above inequalities, form a deformed octahedral and are separable states. In the other four regions one of the above inequality will not hold, therefore they represent entangled states. Bellow we consider entangled states corresponding to the violation of inequality (4-23) i.e. the states which satisfy the following inequality

$$
\left(p_{1}-p_{2}\right)>\sqrt{4 p_{3} p_{4} / \sin ^{2} 2 \theta+\left(p_{3}-p_{4}\right)^{2}} .
$$

Now in order to obtain optimal LSD of entangled Iso-concurrence decomposable state given in $(4-1)$, with $\left(p_{1}-p_{2}\right)>\sqrt{4 p_{3} p_{4} / \sin ^{2} 2 \theta+\left(p_{3}-p_{4}\right)^{2}}$, we first choose an arbitrary separable state

$$
\rho_{s}^{\prime}=\sum_{i=1}^{4} p_{i}^{\prime}\left|\phi_{i}\right\rangle\left\langle\phi_{i}\right|, \quad 0 \leq p_{i} \leq 1, \quad \sum_{i=1}^{4} p_{i}^{\prime}=1, \quad p_{1}^{\prime}<p_{2}^{\prime}+\sqrt{4 p_{3}^{\prime} p_{4}^{\prime} / \sin ^{2} 2 \theta+\left(p_{3}^{\prime}-p_{4}^{\prime}\right)^{2}}
$$


in the separable region. Then using strict SDP optimization prescription of section (2), we try to optimize $\operatorname{Tr}\left(\Lambda \rho_{s}^{\prime}\right)$ with respect to $\rho-\Lambda \rho_{s}^{\prime}>0$, where the feasible solution corresponds to

$$
\Lambda_{\max }=\min \left\{\frac{p_{1}}{p_{1}^{\prime}}, \frac{p_{2}}{p_{2}^{\prime}}, \frac{p_{3}}{p_{3}^{\prime}}, \frac{p_{4}}{p_{4}^{\prime}}\right\}
$$

Now, using the inequalities

$$
\Lambda_{\max } \leq \frac{p_{i}}{p_{i}^{\prime}}, \text { for } i=2,3,4
$$

and summing over the indices $\mathrm{i}=2,3$ and 4 , we obtain

$$
\left(1-p_{1}^{\prime}\right) \Lambda_{\max } \leq\left(1-p_{1}\right)
$$

since, we have

$$
\frac{p_{1}}{p_{1}^{\prime}} \geq \frac{1-p_{1}}{1-p_{1}^{\prime}}, p_{1}>p_{2}+\sqrt{4 p_{3} p_{4} / \sin ^{2} 2 \theta+\left(p_{3}-p_{4}\right)^{2}} \geq p_{1}^{\prime} .
$$

The only possible choice of $\Lambda_{\max }$ which is consistent with the positivity of $\rho-\Lambda_{\max } \rho_{s}^{\prime}$ is

$$
\Lambda_{\max }=\frac{\left(1-p_{1}\right)}{\left(1-p_{1}^{\prime}\right)} .
$$

This choice of $\Lambda_{\max }$ given in (4-33) saturates the inequalities (4-30) and turns the inequalities to equalities, that is, we have $p_{i}^{\prime}=\frac{p_{i}}{\Lambda_{\max }}, i=2,3,4$. The equation (4-33) indicates that $\Lambda_{\max }$ is a monotonic increasing function of $p_{1}^{\prime}$ and its maximum value corresponds to

$$
p_{1}^{\prime}=p_{2}^{\prime}+\sqrt{4 p_{3}^{\prime} p_{4}^{\prime} / \sin ^{2} 2 \theta+\left(p_{3}^{\prime}-p_{4}^{\prime}\right)^{2}}
$$

with

$$
\Lambda_{\max }=\frac{1-p_{1}}{1-p_{1}^{\prime}}
$$

and

$$
p_{i}^{\prime}=\frac{p_{i}\left(1-p_{1}^{\prime}\right)}{\left(1-p_{1}\right)}, i=2,3,4 .
$$

According to relation (4-34) we have

$$
\Lambda_{\text {max }}=1-\left(p_{1}-p_{2}\right)+\sqrt{4 p_{3} p_{4} / \sin ^{2} 2 \theta+\left(p_{3}-p_{4}\right)^{2}} .
$$


Substituting the results that obtained for $\Lambda_{\max }$ and $p_{i}^{\prime}, i=1,2,3,4$ in $\rho-\Lambda_{\max } \rho_{s}^{\prime}$, we obtain

$$
\rho-\Lambda_{\max } \rho_{s}^{\prime}=\frac{C}{\sin 2 \theta}\left|\phi_{1}\right\rangle\left\langle\phi_{1}\right|
$$

( $C$ is concurrence defined in [15]) which is a pure state in agreement with theorem (2) of Ref.[3].

Therefore, we have

$$
\rho=\left(1-\left(p_{1}-p_{2}\right)+\sqrt{4 p_{3} p_{4} / \sin ^{2} 2 \theta+\left(p_{3}-p_{4}\right)^{2}}\right) \rho_{s}^{\prime}+\left(2 p_{1}-1\right)\left|\phi_{1}\right\rangle\left\langle\phi_{1}\right|,
$$

which is optimal LSD of ICD states in agreement with [5].

In the special case of $(\theta=\pi / 4)$ we obtain Bell decomposable state and $\Lambda_{\max }=2\left(1-p_{1}\right)$.

\subsection{A generic $2 \times 2$ density matrix in Wootters's basis}

In this subsection we obtain optimal LSD for a generic two qubit density matrix by using Wootters basis. Wootters in [19] has shown that for any two qubit density matrix $\rho$ there always exist a decomposition

$$
\rho=\sum_{i}\left|x_{i}\right\rangle\left\langle x_{i}\right|
$$

called Wootters's basis, such that

$$
\left\langle x_{i} \mid \tilde{x}_{j}\right\rangle=\lambda_{i} \delta_{i j}
$$

where $\lambda_{i}$ are square roots of eigenvalues, in decreasing order, of the non-Hermitian matrix $\rho \tilde{\rho}$ and

$$
\tilde{\rho}=\left(\sigma_{y} \otimes \sigma_{y}\right) \rho^{*}\left(\sigma_{y} \otimes \sigma_{y}\right)
$$

where $\rho^{*}$ is the complex conjugate of $\rho$ when it is expressed in a standard basis such as $\{|\uparrow \uparrow\rangle,|\uparrow \downarrow\rangle,|\downarrow \uparrow\rangle,|\downarrow \downarrow\rangle\}$ and $\sigma_{y}$ represent Pauli matrix in local basis $\{|\uparrow\rangle,|\downarrow\rangle\}$. Based on this, the concurrence of the mixed state $\rho$ is defined by $\max \left(0, \lambda_{1}-\lambda_{2}-\lambda_{3}-\lambda_{4}\right)$ [19].

Now let us define states $\left|x_{i}^{\prime}\right\rangle$ as

$$
\left|x_{i}\right\rangle=\frac{\left|x_{i}^{\prime}\right\rangle}{\sqrt{\lambda_{i}}}, \quad \text { for } i=1,2,3,4 .
$$


Then $\rho$ can be expanded as

$$
\rho=\sum_{i} \lambda_{i}\left|x_{i}^{\prime}\right\rangle\left\langle x_{i}^{\prime}\right|
$$

and Eq. (4-41) takes the following form

$$
\left\langle x_{i}^{\prime} \mid \tilde{x}_{j}^{\prime}\right\rangle=\delta_{i j}
$$

Also Wootters has shown that $\rho$ is separable if $\lambda_{1}-\lambda_{2}-\lambda_{3}-\lambda_{4} \leq 0$ and if $\rho$ is in boundary separable state then $\lambda_{1}=\lambda_{2}+\lambda_{3}+\lambda_{4}$. By defining $P_{i}=\lambda_{i} K_{i}$, where $k_{i}=\left\langle x_{i}^{\prime} \mid x_{i}^{\prime}\right\rangle$, then normalization condition of $\rho$ leads to

$$
\operatorname{Tr}(\rho)=\sum_{i=1}^{4} P_{i}=1
$$

Now in order to obtain optimal LSD of entangled Wootters state given in (4-40), (4-41), with $\lambda_{1}>\lambda_{2}+\lambda_{3}+\lambda_{4}$, we first choose an arbitrary separable state

$$
\rho_{s}^{\prime}=\sum_{i=1}^{4} \lambda_{i}^{\prime}\left|x_{i}^{\prime}\right\rangle\left\langle x_{i}^{\prime}\right|, \quad 0 \leq \lambda_{i}^{\prime} \leq \frac{1}{k_{i}}, \quad \sum_{i=1}^{4} \lambda_{i}^{\prime} k_{i}=1, \quad \lambda_{1}^{\prime}<\lambda_{2}^{\prime}+\lambda_{3}^{\prime}+\lambda_{4}^{\prime} .
$$

in the separable region. Then using strict SDP optimization prescription of section (2), we try to optimize $\operatorname{Tr}\left(\Lambda \rho_{s}^{\prime}\right)$ with respect to $\rho-\Lambda \rho_{s}^{\prime}>0$, where the feasible solution corresponds to

$$
\Lambda_{\max }=\min \left\{\frac{\lambda_{1}}{\lambda_{1}^{\prime}}, \frac{\lambda_{2}}{\lambda_{2}^{\prime}}, \frac{\lambda_{3}}{\lambda_{3}^{\prime}}, \frac{\lambda_{4}}{\lambda_{4}^{\prime}}\right\}=\min \left\{\frac{p_{1}}{p_{1}^{\prime}}, \frac{p_{2}}{p_{2}^{\prime}}, \frac{p_{3}}{p_{3}^{\prime}}, \frac{p_{4}}{p_{4}^{\prime}}\right\}
$$

Now, using the inequalities

$$
\Lambda_{\max } \leq \frac{p_{i}}{p_{i}^{\prime}}, \text { for } i=2,3,4
$$

and summing over the indices $\mathrm{i}=2,3$ and 4 , we obtain

$$
\left(1-p_{1}^{\prime}\right) \Lambda_{\max } \leq\left(1-p_{1}\right)
$$

since, we have

$$
\frac{\lambda_{1}}{\lambda^{\prime}} \geq \frac{1-\lambda_{1}}{1-\lambda_{1}^{\prime}}, \lambda_{1}>\lambda_{2}^{\prime}+\lambda_{3}^{\prime}+\lambda_{4}^{\prime} \geq \lambda_{1}^{\prime}
$$


The only possible choice of $\Lambda_{\max }$ which is consistent with the positivity of $\rho-\Lambda_{\max } \rho_{s}^{\prime}$ is

$$
\Lambda_{\max }=\frac{\left(1-p_{1}\right)}{\left(1-p_{1}^{\prime}\right)}=\frac{\left(1-k_{1} \lambda_{1}\right)}{\left(1-k_{1} \lambda_{1}^{\prime}\right)} .
$$

This choice of $\Lambda_{\max }$ given in (4-52) saturates the inequalities (4-49) and turns the inequalities to equalities, that is, we have $\lambda_{i}^{\prime}=\frac{\lambda_{i}}{\Lambda_{\max }}, i=2,3,4$. The equation (4-52) indicates that $\Lambda_{\max }$ is a monotonic increasing function of $\lambda_{1}^{\prime}$ and its maximum value corresponds to

$$
\lambda_{1}^{\prime}=\lambda_{2}^{\prime}+\lambda_{3}^{\prime}+\lambda_{4}^{\prime}
$$

with

$$
\Lambda_{\max }=\frac{1-\lambda_{1} k_{1}}{1-\lambda_{1}^{\prime} k_{1}}
$$

and

$$
\begin{gathered}
\lambda_{i}^{\prime}=\frac{\lambda_{i}\left(1-\lambda_{1}^{\prime} k_{1}\right)}{1-\lambda_{1} k_{1}}, \\
\lambda_{1}^{\prime}=\frac{\left(\lambda_{2}+\lambda_{3}+\lambda_{4}\right)}{1-\lambda_{1} k_{1}} .
\end{gathered}
$$

Therefore, we can show that

$$
\Lambda_{\max }=1-k_{1}\left(\lambda_{1}-\lambda_{2}-\lambda_{3}-\lambda_{4}\right)=1-k_{1} C
$$

where $C$ is concurrence. Using $k_{i}=\left\langle x_{i}^{\prime} \mid x_{i}^{\prime}\right\rangle$ and (4-43) we obtain

$$
\Lambda_{\max }=1-\frac{C}{\lambda_{1}}\left\langle x_{1} \mid x_{1}\right\rangle .
$$

Substituting the results that obtained for $\Lambda_{\max }$ and $\lambda_{i}^{\prime}, i=1,2,3,4$ in $\rho-\Lambda_{\max } \rho_{s}^{\prime}$, we obtain

$$
\rho-\Lambda_{\max } \rho_{s}^{\prime}=C\left|x_{1}^{\prime}\right\rangle\left\langle x_{1}^{\prime}\right|
$$

which is a pure states in agreement with theorem (2) of Ref.[3] .

Therefore, we have

$$
\rho=\left(1-\frac{C}{\lambda_{1}}\left\langle x_{1} \mid x_{1}\right\rangle\right) \rho_{s}^{\prime}+C\left|x_{1}^{\prime}\right\rangle\left\langle x_{1}^{\prime}\right|,
$$

which is optimal LSD of states in agreement with [5]. 


\section{4 $2 \otimes 3$ Bell decomposable state}

In this subsection we obtain optimal LSD for the Bell decomposable states of $2 \otimes 3$ quantum systems. A Bell decomposable density matrix acting on $2 \otimes 3$ Hilbert space can be defined by

$$
\rho=\sum_{i=1}^{6} p_{i}\left|\psi_{i}\right\rangle\left\langle\psi_{i}\right|, \quad 0 \leq p_{i} \leq 1, \quad \sum_{i=1}^{6} p_{i}=1
$$

where $\left|\psi_{i}\right\rangle$ are Bell states in $H^{6} \cong H^{2} \otimes H^{3}$ Hilbert space, defined by:

$$
\begin{aligned}
\left|\psi_{1}\right\rangle & =\frac{1}{\sqrt{2}}(|11\rangle+|22\rangle), & \left|\psi_{2}\right\rangle & =\frac{1}{\sqrt{2}}(|11\rangle-|22\rangle), \\
\left|\psi_{3}\right\rangle & =\frac{1}{\sqrt{2}}(|12\rangle+|23\rangle), & \left|\psi_{4}\right\rangle & =\frac{1}{\sqrt{2}}(|12\rangle-|23\rangle), \\
\left|\psi_{5}\right\rangle & =\frac{1}{\sqrt{2}}(|13\rangle+|21\rangle), & \left|\psi_{6}\right\rangle & =\frac{1}{\sqrt{2}}(|13\rangle-|21\rangle) .
\end{aligned}
$$

It is quite easy to see that the above states are orthogonal and hence they can span the Hilbert space of $2 \otimes 3$ systems. From Peres-Horodeckis $[17,18]$ criterion for separability we deduce that the state given in Eq. (4-61) is separable if and only if the following inequalities are satisfied

$$
\begin{aligned}
& \left(p_{1}-p_{2}\right)^{2} \leq\left(p_{3}+p_{4}\right)\left(p_{5}+p_{6}\right), \\
& \left(p_{3}-p_{4}\right)^{2} \leq\left(p_{5}+p_{6}\right)\left(p_{1}+p_{2}\right), \\
& \left(p_{5}-p_{6}\right)^{2} \leq\left(p_{1}+p_{2}\right)\left(p_{3}+p_{4}\right) .
\end{aligned}
$$

In the following we always assume without loss of generality that $p_{1} \geq p_{2}, p_{3} \geq p_{4}$ and $p_{5} \geq p_{6}$.

Again in order to obtain optimal LSD of entangled BD state given in (4-61), with $p_{1}>$ $p_{2}+\sqrt{\left(p_{3}+p_{4}\right)\left(p_{5}+p_{6}\right)}$, we first choose an arbitrary separable state

$$
\rho_{s}^{\prime}=\sum_{i=1}^{6} p_{i}^{\prime}\left|\phi_{i}\right\rangle\left\langle\phi_{i}\right|, \quad 0 \leq p_{i}^{\prime} \leq 1, \quad \sum_{i=1}^{6} p_{i}^{\prime}=1, \quad p_{1}^{\prime}<p_{2}^{\prime}+\sqrt{\left(p_{3}^{\prime}+p_{4}^{\prime}\right)\left(p_{5}^{\prime}+p_{6}^{\prime}\right)}
$$

in the separable region. Then using strict SDP optimization prescription of section (2), we try to optimize $\operatorname{Tr}\left(\Lambda \rho_{s}^{\prime}\right)$ with respect to $\rho-\Lambda \rho_{s}^{\prime}>0$, where the feasible solution corresponds to

$$
\Lambda_{\max }=\min \left\{\frac{p_{1}}{p_{1}^{\prime}}, \frac{p_{2}}{p_{2}^{\prime}}, \frac{p_{3}}{p_{3}^{\prime}}, \frac{p_{4}}{p_{4}^{\prime}}, \frac{p_{5}}{p_{5}^{\prime}}, \frac{p_{6}}{p_{6}^{\prime}}\right\}
$$


Now, using the inequalities

$$
\Lambda_{\max } \leq \frac{p_{i}}{p_{i}^{\prime}}, \quad \text { for } i=2, \ldots, 6
$$

and summing over the indices $i=2, \ldots$ and 6 , we obtain

$$
\left(1-p_{1}^{\prime}\right) \Lambda_{\max } \leq\left(1-p_{1}\right)
$$

since, we have

$$
\frac{p_{1}}{p_{1}^{\prime}} \geq \frac{1-p_{1}}{1-p_{1}^{\prime}}, p_{1}>p_{2}+\sqrt{\left(p_{3}+p_{4}\right)\left(p_{5}+p_{6}\right)}, p_{1}^{\prime}<p_{2}^{\prime}+\sqrt{\left(p_{3}^{\prime}+p_{4}^{\prime}\right)\left(p_{5}^{\prime}+p_{6}^{\prime}\right)}
$$

The only possible choice of $\Lambda_{\max }$ which is consistent with the positivity of $\rho-\Lambda_{\max } \rho_{s}^{\prime}$ is

$$
\Lambda_{\max }=\frac{\left(1-p_{1}\right)}{\left(1-p_{1}^{\prime}\right)}
$$

This choice of $\Lambda_{\max }$ given in (4-71) saturates the inequalities (4-68) and turns the inequalities to equalities, that is, we have $p_{i}^{\prime}=\frac{p_{i}}{\Lambda_{\max }}, i=2, \ldots, 6$. The equation (4-71) indicates that $\Lambda_{\max }$ is a monotonic increasing function of $p_{1}^{\prime}$ and its maximum value corresponds to $p_{1}^{\prime}=$ $p_{2}^{\prime}+\sqrt{\left(p_{3}^{\prime}+p_{4}^{\prime}\right)\left(p_{5}^{\prime}+p_{6}^{\prime}\right)}$, with

$$
\Lambda_{\text {max }}=\frac{\left(1-p_{1}\right)}{\left(1-p_{1}^{\prime}\right)}=1-p_{1}+p_{2}+\sqrt{\left(p_{3}+p_{4}\right)\left(p_{5}+p_{6}\right)} .
$$

and

$$
p_{i}^{\prime}=\frac{p_{i}}{\Lambda_{\max }}, i=2, \ldots, 6
$$

Substituting the results that obtained for $\Lambda_{\max }$ and $p_{i}^{\prime}, i=1, \ldots, 6$ in $\rho-\Lambda_{\max } \rho_{s}^{\prime}$, we obtain

$$
\rho-\Lambda_{\max } \rho_{s}^{\prime}=\left(1-\Lambda_{\max }\right)\left|\phi_{1}\right\rangle\left\langle\phi_{1}\right|
$$

which is a pure states in agreement with theorem (2) of Ref.[3].

Therefore, we have

$$
\rho=\left(1-p_{1}+p_{2}+\sqrt{\left(p_{3}+p_{4}\right)\left(p_{5}+p_{6}\right)}\right) \rho_{s}^{\prime}+\left(p_{1}-p_{2}-\sqrt{\left(p_{3}+p_{4}\right)\left(p_{5}+p_{6}\right)}\right)\left|\phi_{1}\right\rangle\left\langle\phi_{1}\right|
$$

which is optimal LSD of $2 \times 3$, BD states. 
The above choice of $\Lambda_{\max }$ do not cover the whole set of separable states lying at boundary $p_{1}=p_{2}+\sqrt{\left(p_{3}+p_{4}\right)\left(p_{5}+p_{6}\right)}$. Hence we should try other possible values of $\Lambda_{\max }$ as follows:

$$
p_{i}^{\prime} \Lambda_{\max } \leq p_{i}, i=1,4,5,6
$$

Summing over indices $i=1,2, \ldots, 4$ we obtain

$$
\Lambda_{\max } \leq \frac{1-p_{2}-p_{3}}{1-p_{2}^{\prime}-p_{3}^{\prime}} .
$$

Therefore, maximum feasible choice of $\Lambda_{\max }$ is

$$
\Lambda_{\max }=\frac{1-p_{2}-p_{3}}{1-p_{2}^{\prime}-p_{3}^{\prime}}
$$

which is possible if we choose

$$
p_{i}^{\prime}=\frac{p_{i}}{\Lambda_{\max }}, i=1,4,5,6,
$$

Now substituting $p_{i}^{\prime}=\frac{p_{i}}{\Lambda_{\text {max }}}, i=1,4,5,6$ in normalization condition $\operatorname{Tr}\left(\rho_{s}^{\prime}\right)=\sum_{i=1}^{6}=1$ and the separability equation (4-63) we can solve $\Lambda_{\max }$ as a function of single variable $p_{3}^{\prime}$ and after optimizing it with respect to $p_{3}^{\prime}$ we get

$$
\Lambda_{\max }=1-\left(p_{2}-p_{1}\right)-\left(p_{3}+p_{4}\right)-\frac{1}{4}\left(p_{5}+p_{6}\right)
$$

and

$$
p_{2}^{\prime}=\frac{2 p_{1}-p_{5}-p_{6}}{2 \Lambda_{\max }} \quad, p_{3}^{\prime}=\frac{p_{5}+p_{6}-4 p_{4}}{4 \Lambda_{\max }} .
$$

Similarly, following the above procedure for other possible choices, such as

$$
\Lambda_{\max }=\frac{p_{i}}{p_{i}^{\prime}}, i=1,3,4,6
$$

yields

$$
\begin{gathered}
p_{2}^{\prime}=\frac{2 p_{1}-p_{3}-p_{4}}{2 \Lambda_{\max }}, p_{5}^{\prime}=\frac{p_{3}+p_{4}-4 p_{6}}{4 \Lambda_{\max }} . \\
\Lambda_{\max }=1-\left(p_{2}-p_{1}\right)-\left(p_{5}+p_{6}\right)-\frac{1}{4}\left(p_{3}+p_{4}\right) .
\end{gathered}
$$

Of course, using this procedure we can obtain an optimal separate decomposition with rank-3 entangled part of some particular given density matrices. 


\subsection{Werner states}

The Werner states are the only states that are invariant under local unitary operations. For $d \otimes d$ systems the Werner states are defined by $[20]$

$$
\rho_{f}=\frac{1}{d^{3}-d}((d-f) I+(d f-1) F), \quad-1 \leq f \leq 1
$$

where $I$ stands for identity operator and $F=\sum_{i, j}|i j\rangle\langle j i|$. It is shown that Werner state is separable iff $0 \leq f \leq 1$

Now in order to obtain optimal LSD of entangled Werner state given in (4-86), with $-1<$ $f<0$, we first choose an arbitrary separable state

$$
\rho_{s}^{\prime}=\rho_{f}^{\prime}=\frac{1}{d^{3}-d}\left(\left(d-f^{\prime}\right) I+\left(d f^{\prime}-1\right) F\right), \quad 0 \leq f^{\prime} \leq 1
$$

in the separable region. Then using strict SDP optimization prescription of section (2), we try to optimize $\operatorname{Tr}\left(\Lambda \rho_{s}^{\prime}\right)$ with respect to $\rho-\Lambda \rho_{s}^{\prime}>0$, where the feasible solution corresponds to

$$
\Lambda_{\max }=\min \left\{\frac{(f+1)}{\left(f^{\prime}+1\right)}, \frac{(1-f)}{\left(1-f^{\prime}\right)}\right\}=\frac{(f+1)}{\left(f^{\prime}+1\right)}
$$

The equation (4-93) indicates that $\Lambda_{\max }$ is a monotonic increasing function of $f^{\prime}$ and its maximum value corresponds to $f^{\prime}=0$, with

$$
\Lambda_{\max }=f+1
$$

Substituting the results that obtained for $\Lambda_{\max }$ in $\rho-\Lambda_{\max } \rho_{s}^{\prime}$, we obtain

$$
\rho-\Lambda_{\max } \rho_{s}^{\prime}=\rho_{\{f\}}-\Lambda_{\max }(1+f) \rho_{\{f=0\}}=f\left(\frac{F-I}{d^{2}-d}\right),
$$

which is a pure states in agreement with theorem (2) of Ref.[3].

Therefore, we have

$$
\rho_{\{f\}}=(1+f) \rho_{\{f=0\}}+f\left(\frac{F-I}{d^{2}-d}\right) .
$$

which is optimal LSD of Werner states. 


\subsection{Isotropic states}

The $d \otimes d$ bipartite isotropic states are the only ones that are invariant under $U \otimes U^{*}$ operations, where * denotes complex conjugation. The isotropic states of $d \otimes d$ systems are defined by [21]

$$
\rho_{F}=\frac{1-F}{d^{2}-1}\left(I-\left|\psi^{+}\right\rangle\left\langle\psi^{+}\right|\right)+F\left|\psi^{+}\right\rangle\left\langle\psi^{+}\right|, \quad 0 \leq F \leq 1
$$

where $\left|\psi^{+}\right\rangle=\frac{1}{\sqrt{d}} \sum_{i}|i i\rangle$ is maximally entangled state. It is shown that isotropic state is separable when $0 \leq F \leq \frac{1}{d}[21]$.

Now in order to obtain optimal LSD of entangled Isotropic state given in (4-92), with $\frac{1}{d}<f<1$, we first choose an arbitrary separable state

$$
\rho_{s}^{\prime}=\rho_{F}^{\prime}=\frac{1-F^{\prime}}{d^{2}-1}\left(I-\left|\psi^{+}\right\rangle\left\langle\psi^{+}\right|\right)+F^{\prime}\left|\psi^{+}\right\rangle\left\langle\psi^{+}\right|, \quad 0 \leq F^{\prime} \leq \frac{1}{d}
$$

in the separable region. Then using strict SDP optimization prescription of section (2), we try to optimize $\operatorname{Tr}\left(\Lambda \rho_{s}^{\prime}\right)$ with respect to $\rho-\Lambda \rho_{s}^{\prime}>0$, where the feasible solution corresponds to

$$
\Lambda_{\max }=\min \left\{\frac{(F)}{\left(F^{\prime}\right)}, \frac{(1-F)}{\left(1-F^{\prime}\right)}\right\}=\frac{(1-F)}{\left(1-F^{\prime}\right)} \text {. }
$$

The equation (4-93) indicates that $\Lambda_{\max }$ is a monotonic increasing function of $F^{\prime}$ and its maximum value corresponds to $F^{\prime}=\frac{1}{d}$, with

$$
\Lambda_{\max }=\frac{d(1-F)}{(d-1)} .
$$

Substituting the results that obtained for $\Lambda_{\max }$ in $\rho-\Lambda_{\max } \rho_{s}^{\prime}$, we obtain

$$
\rho_{F}-\Lambda_{\max } \rho_{1 / d}=\left(1-\Lambda_{\max }\right)\left|\psi^{+}\right\rangle\left\langle\psi^{+}\right|
$$

which is a pure states in agreement with theorem (2) of Ref.[3].

Therefore, we have

$$
\rho_{F}=\frac{(1-F)}{\left(1-F^{\prime}\right)} \rho_{1 / d}+\left(1-\Lambda_{\max }\right)\left|\psi^{+}\right\rangle\left\langle\psi^{+}\right|
$$

which is optimal LSD of isotropic states. 
Best separable approximation

\subsection{One parameter $3 \otimes 3$ state}

Let us consider a one parameter state acting on $H^{9} \cong H^{3} \otimes H^{3}$ Hilbert space as [13]

$$
\rho_{\alpha}=\frac{2}{7}\left|\psi^{+}\right\rangle\left\langle\psi^{+}\right|+\frac{\alpha}{7} \sigma_{+}+\frac{5-\alpha}{7} \sigma_{-}, \quad 2 \leq \alpha \leq 5
$$

where

$$
\begin{aligned}
& \left|\psi^{+}\right\rangle=\frac{1}{\sqrt{3}}(|11\rangle+|22\rangle+|33\rangle), \\
& \sigma_{+}=\frac{1}{3}(|12\rangle\langle 12|| 23\rangle\langle 23|+| 31\rangle\langle 31|), \\
& \sigma_{-}=\frac{1}{3}(|21\rangle\langle 21|| 32\rangle\langle 32|+| 13\rangle\langle 13|) .
\end{aligned}
$$

$\rho_{\alpha}$ is separable iff $2 \leq \alpha \leq 3$, it is bound entangled iff $3 \leq \alpha \leq 4$ and it is distillable entangled state iff $4 \leq \alpha \leq 5[13]$.

Now in order to obtain optimal LSD of entangled one parameter $3 \otimes 3$ state given in (4-97), with $3<\alpha<5$, we first choose an arbitrary separable state

$$
\rho_{s}^{\prime}=\rho_{\alpha^{\prime}}=\frac{2}{7}\left|\psi^{+}\right\rangle\left\langle\psi^{+}\right|+\frac{\alpha^{\prime}}{7} \sigma_{+}+\frac{5-\alpha^{\prime}}{7} \sigma_{-}, \quad 2 \leq \alpha^{\prime} \leq 3,
$$

in the separable region. Then using strict SDP optimization prescription of section (2), we try to optimize $\operatorname{Tr}\left(\Lambda \rho_{s}^{\prime}\right)$ with respect to $\rho-\Lambda \rho_{s}^{\prime}>0$, where the feasible solution corresponds to

$$
\Lambda_{\max }=\frac{5-\alpha}{5-\alpha^{\prime}}
$$

The equation (4-100) indicates that $\Lambda_{\max }$ is a monotonic increasing function of $\alpha^{\prime}$ and its maximum value corresponds to $\alpha^{\prime}=3$, with

$$
\Lambda_{\max }=\frac{5-\alpha}{2}
$$

Substituting the results that obtained for $\Lambda_{\max }$ in $\rho-\Lambda_{\max } \rho_{s}^{\prime}$, we obtain

$$
\rho-\Lambda_{\max } \rho_{\alpha=3}=\left(2 / 7\left|\psi^{+}\right\rangle\left\langle\psi^{+}\right|+5 / 7 \sigma_{+}\right)
$$

which is a pure states in agreement with theorem (2) of Ref.[3].

Therefore, we have

$$
\rho=\left(\frac{5-\alpha}{2}\right) \rho_{\alpha=3}+\left(2 / 7\left|\psi^{+}\right\rangle\left\langle\psi^{+}\right|+5 / 7 \sigma_{+}\right)
$$

which is optimal LSD of one parameter $3 \otimes 3$ states. 


\subsection{Multi partite isotropic states}

In this subsection we obtain the optimal LSD for a n-partite d-levels system. Let us consider the following mixture of the completely random state $\rho_{0}=I / d^{n}$ and the maximally entangled state $\left|\psi^{+}\right\rangle$

$$
\rho(s)=(1-s) \frac{I}{d^{n}}+s\left|\psi^{+}\right\rangle\left\langle\psi^{+}\right|, \quad 0 \leq s \leq 1
$$

where $I$ denotes identity operator in $d^{n}$-dimensional Hilbert space and $\left|\psi^{+}\right\rangle=\frac{1}{\sqrt{d}} \sum_{i=1}^{d}|i i \cdots i\rangle$. The separability properties of the state (4-104) is considered in Ref. [22]. It is shown that the above state is separable iff $s \leq s_{0}=\left(1+d^{n-1}\right)^{-1}$.

Now in order to obtain optimal LSD of entangled multi partite isotropic state given in (4-104), with $s_{0}<s<1$, we first choose an arbitrary separable state

$$
\rho_{s}^{\prime}=\rho\left(s^{\prime}\right)=\left(1-s^{\prime}\right) \frac{I}{d^{n}}+s^{\prime}\left|\psi^{+}\right\rangle\left\langle\psi^{+}\right|, \quad 0 \leq s^{\prime} \leq s_{0}
$$

in the separable region. Then using strict SDP optimization prescription of section (2), we try to optimize $\operatorname{Tr}\left(\Lambda \rho_{s}^{\prime}\right)$ with respect to $\rho-\Lambda \rho_{s}^{\prime}>0$, where the feasible solution corresponds to

$$
\Lambda_{\max }=\frac{1-s}{1-s^{\prime}}
$$

The equation (4-106) indicates that $\Lambda_{\max }$ is a monotonic increasing function of $s^{\prime}$ and its maximum value corresponds to $s^{\prime}=s_{0}$, with

$$
\Lambda_{\max }=\frac{1-s}{1-s_{0}}=\frac{(1-s)\left(1+d^{n-1}\right)}{d^{n-1}} .
$$

Substituting the results that obtained for $\Lambda_{\max }$ in $\rho-\Lambda_{\max } \rho_{s}^{\prime}$, we obtain

$$
\rho(s)-\Lambda_{\max } \rho\left(s=s_{0}\right)=\left(1-\Lambda_{\max }\right)\left|\psi^{+}\right\rangle\left\langle\psi^{+}\right|
$$

which is a pure states in agreement with theorem (2) of Ref.[3].

Therefore, we have

$$
\rho(s)=\frac{(1-s)\left(1+d^{n-1}\right)}{d^{n-1}} \rho\left(s=s_{0}\right)+\left(1-\frac{(1-s)\left(1+d^{n-1}\right)}{d^{n-1}}\right)\left|\psi^{+}\right\rangle\left\langle\psi^{+}\right|
$$

which is optimal LSD of multi partite isotropic states. 


\section{Conclusion}

Here in this work we have been able to obtain LSD of bunch of mixed state density matrices via an elegant method of convex positive semidefinite optimization methods, where the results that obtained are in agreement with those obtained by other methods in Ref.[14, 15]. Comparing this method with those of previously introduced one, one can appreciate the elegance and usefulness of SDP method in connection with LSD.

\section{References}

[1] A. Einstein, B. Podolsky and Rosen, Phys. Rev. 47, 777 (1935).

[2] E. Schrödinger, Naturwissenschaften. 23, 807 (1935).

[3] M. Lewenstein and A. Sanpera, Phys. Rev. Lett. 80, 2261 (1998).

[4] B. G Englert and N. Metwally, J. Mod. Opt. 47, 2221 (2000).

[5] T. Wellens and M. Kus, Phys. Rev. A 64, 022303 (2001).

[6] S. Karnas and M. Lewenstein, J. Phys. A: Math. Gen. 34 , 6919 (2001).

[7] E. Rain, IEEE Transactions on Information Theory. 47, 2921 (2001).

[8] F. Verstraete and H. Verschelde, Phys. Rev. Lett. 90, 097901 (2003).

[9] A. C. Doherty, P. A. Parrilo and F. M. Spedalieri, Phys. Rev. A 69, 022308 (2004).

[10] L. Vandenberghe and S. Boyd, SIAM Review. 38, 49 (1996).

[11] S. Boyd and L. Vandenberghe, Convex Optimization, Cambridge University Press, (2004).

[12] R. Horodecki and M. Horodecki, Phys. Rev. A 54, 1838 (1996). 
[13] P. Horodecki, M. Horodecki and R. Horodecki, Phys. Rev. Lett. 82, 1056 (1999).

[14] S. J. Akhtarshenas, M. A. Jafarizadeh, Quantum Information and Computation. Vol 3, No. 3, 229 (2003).

[15] S. J. Akhtarshenas, M. A. Jafarizadeh, J. Phys. A: Math. Gen. 37, 2965 (2004)

[16] A. Ericsson, Phys. Lett. A 295, 256 (2002).

[17] A. Peres, Phys. Rev. Lett. 77, 1413 (1996).

[18] M. Horodecki, P. Horodecki and R. Horodecki, Phys. Lett. A 223, 1 (1996).

[19] W. K. Wootters, Phys. Rev. Lett. 80, 2245 (1998).

[20] R. F. Werner, Phys. Rev. A 40, 4277 (1989).

[21] M. Horodecki and P. Horodecki, Phys. Rev. A 59, 4206 (1999).

[22] A. O. Pittenger and M. H. Rubin, Optics Communications. 179, 447 (2000). 